\title{
DOI: 10.7596/taksad.v8i4.2372
}

Citation: Efimova, L. S., \& Pavlova, O. K. (2019). Complex Epithet Constructions: The North-Eastern Yakut Olonkho Telling Tradition. Journal of History Culture and Art Research, 8(4), 229-237. doi:http://dx.doi.org/10.7596/taksad.v8i4.2372

\section{Complex Epithet Constructions: The North-Eastern Yakut Olonkho Telling Tradition}

\author{
Ludmila Stepanovna Efimova ${ }^{1}$, Olga Ksenofontovna Pavlova ${ }^{2}$
}

\begin{abstract}
The present article describes complex epithet constructions, which were distinguished in the north-eastern Yakut Olonkho-telling tradition. The aim of the research is to study complex epithet constructions: epithets of comparison, numerical, and metaphoric epithets. There were a total of 251 (26\%) epithets with complex comparisons found in the text in the course of its analysis. The first group comprises of epithets connected with natural objects. In Olonkho, the descriptions of the northern Yakutia territory (i.e., its plant life, water landscapes, general landscapes, and celestial events) are introduced by incorporating these epithets, which are widely used. The second group of complex epithets with comparisons comprises of somatic epithets which give a general description of the main and secondary characters through the body and appearance description as well as the gestures and emotions. The third group comprises of complex epithets, the objects of which are connected with dwellings, household dwellings, and general household features. A significant meaning of the bogatyr (ancient warrior) horse in the life of the main characters of Yakut Olonkho allows singling out a separate, the fourth group of epithets, i.e., 15 complex epithets, the objects of which are those denoting horse body parts. The north-eastern Olonkho text contained 382 instances of numbers used as component-parts of numerical epithets. The numerical epithets are subdivided into three groups. Thus, the first group, which is the largest among all three (91\%) consists of numerical epithets, which in turn, include linear numerals as well as cardinal numerals and ones denoting age and number adverbs. The second group contains a smaller scope of numerical epithets (7\%); these include numerical epithets based on the usage of doubledigit numbers. The third and the smallest group (2\%) includes numerical epithets which contain threedigit components (2\%). Numerical epithets are one of the widely used epithets in the poetic structure of the epic tradition under study. Apparently, the numerals in this type of epithet constructions performed not only a comprehensive and descriptive but also an educational function. Metaphoric epithets make up $15 \%$ of the total amount of epithets identified and selected within the scope of the north-eastern Olonkho text research. They are equally simple and complex in their essence. Simple metaphoric epithets consist of one object and image. Complex epithets contain several objects, each of which has its own attributive combinations; these attributive combinations consist of a noun in its non-transferred meaning and a metaphor. Thus, epithets represented in the Olonkho texts are complex in their structure (26\%).
\end{abstract}

Keywords: Turkic epic, Yakut epic, olonkho, epic traditions, north-eastern tradition, poetics, epithets, numerical epithets, metaphoric epithets, epithets with comparisons.

\footnotetext{
${ }^{1}$ Doctor of Philological Sciences, Head of the Department of Cultural Studies, Institute of Languages and Cultures of the Peoples of the North-East, North-Eastern Federal University, Yakutsk, Russia. E-mail: ludmilaxoco@mail.ru

${ }^{2}$ Candidate of Philological Sciences, Senior Lecturer, Department of Folklore and Culture Studies, Institute of Languages and Cultures of the Peoples of the North-East, North-Eastern Federal University, Yakutsk, Russia. Email: olga-ksento@mail.ru
} 


\section{Introduction}

A.N. Veselovsky defined epithets as qualities of certain objects noting that "an epithet is a flat definition of a word or its undermined notional meaning or a word signifying and highlighting a unique quality, which is typical for the described object" (Veselovsky, 1940 p.59). B.V. Troshchansky held the opinion that an epithet is, first of all, a feature or a definition of an object. He states the following: "a poetic attribute expresses the feature which is concealed with the attributive word itself, and has the goal of focusing the attention of the listener/reader to this feature; or it expresses the emotional attitude of the speaker towards a specific object. The poetic attribute is called an epithet" (Tomashevsky, 2002 p.58).

F.M. Selivanov distinguished simple, complex and extended epithets in the poetic structure of Russian bylinas. As for extended epithets, the scholar noted that "each consecutive epithet joined with a defining word serves as a clarification or as an epithet to the previous epithet, or a clarification to a combination with an epithet" (Selivanov, 1977, p.102).

A certain time later, in his special studies, A.V. Pavshuk on the special nature of epithets defines an epithet as "a creative attribute of an object, a person, a phenomenon or a process, which signifies (highlights)... a significant feature; this feature can repeat and renew meaning of the identified word, create micro- or macro image, giving it a creative touch; it can also convey hidden meanings and evaluations, set the emotional mood of the storyline, reinforce the impressions of the story recipient, turning to the listener's/reader's intellectual, emotional and esthetic perception" (Pavshuk, 2007, p.20). Epithets introduced in the poetic structure of any story, performed an attributive and specifying function.

Yakut Olonkho has a significant variety of epithets, which give the storyline its festiveness and transfer the main events of the plot into a wonderful and exquisite imaginary world. Epithets were described from different standpoints in the research papers published by the following Yakut scholars: A.A. Ivanov-Kunde (1979), I.V. Pukhov (1962), V.T. Petrov (1978), P.A. Sleptsov (1989), L.N. Romanova (2002), L.S. Efimova (2004), V.G. Semenova (1999), etc. These scholars distinguish the following types of epithets: simple, emphasizing, metaphorical, extended, fixed, evaluative, poetic, "archaic", general folkloric, genre-dependent, explicative, hyperbolic, descriptive, evaluativedescriptive, evaluative-poetic, frequent and trite, numerical and color epithets.

It is worth mentioning that folklore epithets were significant in complex formation and structure. In terms of their components, these epithets comprise of an attributive word and a nucleus word. In terms of their structure as presented in the Yakut folklore genres, they can be simple and complex. For instance, there is a prevailing number of various complex epithets in the Yakut round dance songs (Efimova, 2004, p.14).

For this reason, we have differentiated the object of the epithets (the object of description or attribution) as a methodology of research in the course of folklore epithets analysis. In terms of its grammatical form, this type of epithet is expressed by an attributive word (a term coined by F.M. Selivanov). In the next stage, we singled out the image of an epithet, which characterizes and identifies the special features, shades of qualities of an object as well as the phenomena which are compared to or contrasted with the object of the epithet. The image defined by the nuclei (defining) words. 
It is typical for the north-eastern Olonkho-telling tradition to contain complex epithet constructions. We have identified a total of 251 such epithets which accounts for $26 \%$ of the total amount of epithets. They can be subdivided into three groups: epithets containing comparisons, numerical and metaphoric epithets.

\section{Complex epithets containing comparisons}

It is very typical for this type of poetic structure, which serves as the main focus of this paper, to contain complex epithets with comparisons; this group accounts for $35 \%$ of the overall total of complex epithets which were analyzed. Such epithets, with the exception of the attributive words themselves, include comparisons that are formed by adding the particles kurduk (how) and sagha (just like). In order to simplify the analysis procedure, these epithets containing comparison were differentiated in accordance with the thematic field of the object they describe.

The first group includes epithets of comparison associated with nature representations. They contain 122 epithets, which fall under this group. In general, the north-eastern Olonkho-tellers give a through description of the natural world surroundings (forests, river valleys, trees and herbs). The objects of these epithets are natural resources: trees (tiit - larch tree, hariya - fir-tree, talah shrubs, hatyng - birch-tree, bolbukta - Siberian dwarf pine).

The objects for epithet of comparison, which occur in the Olonkho text, provide a description of nature in the northern part of Yakutia, where the main forest and tree area is covered with larch-trees and shrubs. This abstract from the Momsky region Olonkho text signifies the fact that the Yakut Olonkho of the regions under research in this paper is strictly linked to real-existing lands on the territory of Yakutia: "Kobuo Dzhaghyl", a complex epithet: Sangiyahtaakh omuk ogholoro Tommoru-taary motoruha Sassa turallaryn kurduk, Lobuo hara bolbukta: "The Siberian dwarf pine just like the children of the people from unknown faraway lands" (Muoma olonkholoro, 2004, p.208). The object of this complex epithet is bolbukta - a Siberian dwarf pine which only grows in the sub-mountain areas of northern Yakutia. Due to the fact that the Olonkho-tellers described nature of only the places they knew, the descriptions of an evergreen pine tree and poetic tropes associated with it are typical only for the north-eastern Olonkho-telling tradition.

Water bodies also serve as the objects of poetic inspiration, which allow creating memorable images for the listeners incorporating the epithets of comparison: uu - water, kuol - a lake, along with mountains, mountain hills, clouds, and lakes.

The second thematic group includes not only complex epithets of comparison but also somatisms, which can semantically be identified as those belonging to body parts of the Olonkho characters, including a description of the physical and emotional condition. Using somatism allows the storyteller to provide a colorful depiction of the archaic method of learning about the world surroundings through the physical features of an individual. The analyzed north-eastern Olonkho texts contain 87 somatic epithets.

An external (physical) identification of the main and secondary characters is achieved through the body descriptions (e.g., ilii - a hand, atah - a leg, soto - a hip, etc.), hair and face descriptions (e.g., harakh - eyes, murun - a nose, uos - lips, im - cheeks). For instance, in the Momsky region Olonkho telling a story about Kotor Mulgun (The Flying Mulgun), the gestures and 
reactions of the main character help to clarify the qualitative and evaluative characteristics (Tab. 3.1). Therefore, the use of complex epithets containing a somatic component allowed the Olonkhotellers to thoroughly describe the social and physical characteristics of the main characters (bogatyrs), to highlight their strength, power, and beauty. The third group comprises of the epithet objects, which characterize dwellings, household dwelling, and household utensils and tools. This group contains 85 epithet objects.

They contain a majority of objects, which are connected to the functions or dwelling parts descriptions (e.g., modzhogho - a threshold, kirilies a porch, istiene - walls); and dwelling zones and furniture (e.g., kuruo and olbuor - a fence, tusaha - a yard); furniture (oron - a bed, ostuol - a table, oloh - a chair, etc.), household kitchenware (e.g., nyuosku - a spoon, tiilke - a fork, terielke - a plate).

The north-eastern Olonkho also contains objects, which denote hunting and fighting tools (e.g., saa - an elephant gun, okh - a bow, onoghos - an arrow, annyy - a sword, batyia - a close range battle spear). The majority of fixed comparisons used in the Olonkho connected with various types of hunting tools, indicate that there were main heroes in the epic whose occupation was hunting and. A significant role in the description of the heroic dwellings in the Olonkho world is given to a Yakut hearth called kamelyok; each of its components is described in detail; the pipe (turba) and the perch roost (holumtan) are thoroughly described. Thus, in the Momsky version of Olonkho Kotor Mulgun (Flying Mulgun) the following epithet is used to denote the perch roost: alaas syhyy byhaghahyn sagha kyrday taas sostuok (Lit.: "a stone perch roost the size of a half of a meadow") (Muoma olonkholoro, 2004, p.16).

The fourth group consists of 15 complex epithets of comparison, the object of which is the bogatyr horse. This choice of the epithet object is explained by the fact that the horse plays an exceptional role in the life and destiny of the main character (bogatyr).

The Olonkho Aaliya Berge (The Precise Aaliya) contains 4 additional epithets of comparison, which serve as horse descriptions and has the following components parts: kulgaah (ears), ayakh (a jaw), tanyy (a nostril), and tonoghos (the horse spine).

Verbal constructions belong to such important and main components of the object as kulgaah (ears), tuyah (hoofs), muhe (a hip), tonoghos (a spine), etc. For example, the Verkhoyansky version of Olonkho Aaliya Bergen (the Precise Bergen) provides the following description of the bogatyr horse: Turba uutun kurduk turuoru kulgaahtaakh, Dzhiye ayaghyn sagha dzhellem ayahtaakh, Uoles uutun kurduk ongoruk tanyylaakh, Dulung mas kurduk chirke kono monoghostookh (Lit.: "Ears, like a straight pipe, with a jaw the size of a balaghan door, nostrils the size of a chimney hole, a spine like a log" (Ammosov, 2008, p.22).

Thus, the analyzed texts of the north-eastern version of Olonkho revealed a total of 251 (26\%) epithets of comparison. The first group includes epithets associated with natural objects. With the help of these frequently used epithets in the Olonkho text, there is a description of the northern Yakutia territory unfolding in front of the listener/reader; they describe the plant life, water landscapes, general landscapes and celestial phenomena. The second group of complex epithets of comparison comprises of somatic epithets, with the help of which, the storyteller describes the physical appearance of the characters, their gestures, emotions and there is a general external 
identification of the main and secondary characters. The third group consists of complex epithets, the objects of which are associated with dwellings, household dwellings, and household objects. The bogatyr horse plays a significant role in the life and journeys of the main characters in the Yakut Olonkho and it forms a separate, fourth group comprising of 15 complex epithets, the objects of which are horse body parts.

\section{Numerical epithets}

Epithets, the component of which is a numeral, are called numerical epithets. The popular usage of such epithets can be explained by the necessity of interpreting the sacred meaning of simple numerals in the epics of the Turkic-Mongolic peoples. E.I. Izbekova explains the sacred meaning of epithets used in Yakut Olonkho and states that this is connected with the archaic layers of the ancient Yakut worldview: "The sacred symbolism is typical for epics, which date back to the archaic time-period... For instance, there is sacred symbolism in the epic numeral us (three), sette (seven), aghys (eight), toghus (nine); it originates from the mythological beliefs of the ancient Yakut people" (Izbekova, 2014, p.133).

We suggest subdividing the numerical epithets, which were found in the course of analysis, into three groups.

The first group of these epithets is represented by epithets, which contain the first sequence of cardinal numerals. Interestingly, the Olonkho texts of the tradition discussed in this paper, there is wide use of all the numerals from 1 to 10 . The number us (three) is used in the text 95 times, sette (seven) - used in the text 64 times, aghys (eight) - used in the text 75 times, toghus (nine) mentioned in the text 80 times. Their total percentage amounts for $91 \%$ of the overall total.

The most frequently used number is us (three). These numbers symbolize a range of concepts in the Yakut folklore. We will provide an example from the Olonkho of the Srednekolymsky region: Yuheetten buollar us biis uuhun tyyna tangnary sarkyraan tuhen tobo yaryy buoluo dien - us hos timir urutteeh (Lit.: "A three-layered iron ceiling, so that the breath of the inhabitants of the Upper World would not reach them"). The number three represents the entire world. The Yakut Olonkho of the Abiysky region perceives the number three as the general number of people living in the Aiyy tribe as well as the distance or the size of an object and even the Universe itself. Sometimes, the number three can be translated as "infinite", as in the following example: yus daydynan buoghardaah uktaah, saabylaan batastaah (Lit.: A sword that spread itself over the entire Universe); the object Yus daydy is translated as "the Universe".

Additionally, the numerals include epithets which consist of cardinal numbers with the addition of the following affixes: -aah: aghys+taah (an eight-year-old), toghus+taah (a nine-year-old). They denote the person's or the animal's age.

Next, there are count adverbs, which are formed from the cardinal number with the addition of an affix. The Olonkho texts of the tradition described in this paper contain such count adverbs as: uste (thrice), etc. A.M. Peshkovsky classified these adverbs (twice, thrice) as count adverbs.

The second group, which is less significant in the amount of numerical epithets, includes epithets based on the use of double-digit numbers from 20 to 99 ( $7 \%$ of the overall total). The selection shows that the texts contain numerical epithets based on the use of the following 
numbers: suurbe (twenty), otut (thirty), tuort uon (forty), sette uon (seventy), sette uon sette (seventy-seven), aghys uon (eighty), aghys uon aghys (eighty-eight), toghus uon (ninety), toghus uon toghus (ninety nine). The most commonly used number is otut (thirty) (used 10 times). It is possible that the number otut (thirty), just like three, has a sacred symbolism, as well as the numbers sette uon (seventy), sette uon sette (seventy-seven), aghys uon (eighty), aghys uon aghys (eighty-eight), toghus uon (ninety), toghus uon toghus (ninety-nine).

The third group of numerical epithets includes constructions based on the use of three-digit numbers; it is the smallest group in number (only $2 \%$ ). This group includes epithets formed using the numerals us suus (three hundred), bies suus (five hundred) and aghys suus (eight hundred).

In the texts of the Olonkho of the north-eastern tradition, there are 382 cases of the use of numerals as part of numerical epithets. Numerical epithets are subdivided into three groups. Hence, the first and the largest group (91\%) consists of numerical epithets, which include first-order numerals, including quantitative numerals and numerals that indicate age and count adverbs. The second, a much smaller group of numerical epithets $(7 \%)$ includes numerical epithets based on the use of double-digit numbers. The third, smallest group (2\%) includes numerical epithets the components of which are three-digit numbers (2\%). Numerical epithets are one of the most widely used epithets in the poetics of this epic tradition. Apparently, the numerals in the composition of epithet constructions of this type had not only comprehensive, descriptive, but also educational functions.

\section{Metaphoric epithets}

Metaphorical epithets are figurative epithets. They are based on a metaphorical figurative meaning. The classification criteria are the composition and thematic groups of metaphorical epithets. In terms of composition, metaphorical epithets are distinguished as simple and complex. The first group includes simple metaphorical epithets. For example, aghys saastaah atyr sylgy hahatyn tanichchi byrahpyt aalay komus telaekke (Lit.: "Golden plates (as if) unclenched abdominal fat of an eight-year-old stallion" (Muoma olonholoro, 2004, p.148). In this epithet, the object is the teleche "plate". The object teleekke has its own epithet aalai komus (gold). Next, the object is still endowed with the metaphor aghys saastaah atyr sylgy hahatyn tanichchi byrahpyt "(like) the eightyear-old stallion's flattened abdominal fat", in which the bright yellow (gold) color of the abdominal horse fat, a delicacy in the Yakut traditional cuisine, is transferred to the color of dishes made of precious metal. This comparison in the Yakut language is a metaphor, therefore we propose to consider this type of epithet as metaphorical.

In the following example, urumetiybet uut koluye: (Lit.: "a milk lake (as if) without foam" the object is koluye (lake). Then the object is specified by the epithet uut (dairy), which forms the compound word uut kuleye (milk lake). Before the compound word urumetiybet uut koluye (Lit.: 'a milk lake (as if) without foam" there is a metaphorical epithet urumetiybet ((as if) without foam). So, urumetiybet uut koluye (Lit.: "a milk lake (as if) without foam" is a simple metaphorical epithet.

The second group consists of complex epithets with several attributive structures. The special features of such epithet constructions are that they are characterized by the presence of one object with an image expressed by a complex attributive structure. As an example of the structural 
type of epithet construction, we use the description of the meadow from the text of the Olonkho local tradition (Table 1).

Table 1. Complex epithets with attributive structures

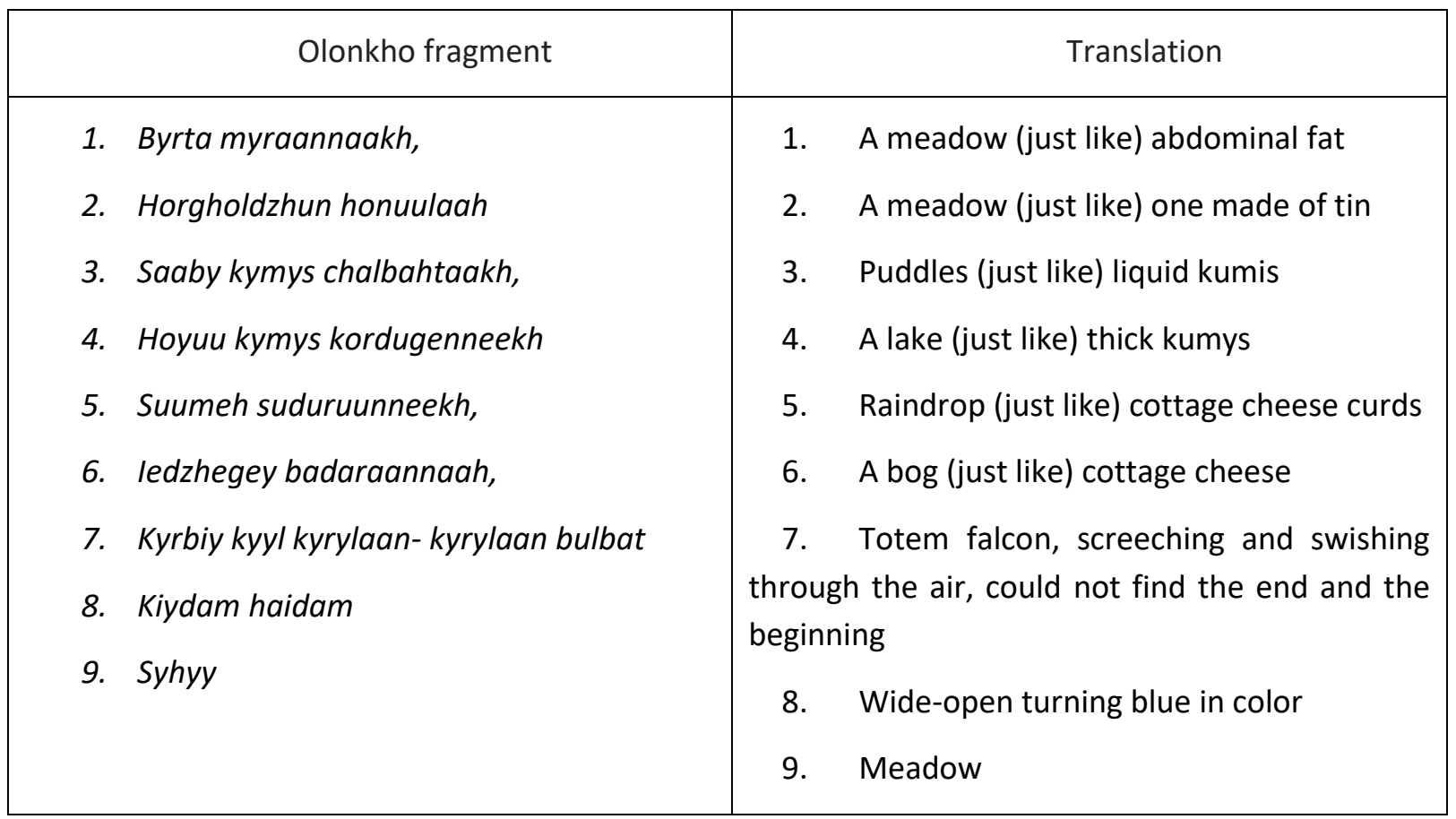

Source: Archives of the Yakutsk Scientific Research Center SB RAS, Fund No.5, storage ID number. 31/109, d.108. p.8. (Initially translated into Russian by the authors of the present article).

The main object of the metaphorical epithet syhyy (a meadow) is indicated by number 9 . The object syhyy (a meadow) and images consisting of simple (7 - 8) and metaphorical epithets (1 - 6) are linked together with comparative relations and formed into an epithet complex using a common comparison indicator (feature) for the compared components. Out of the eight epithets explaining syhyy (a meadow), all six epithets are metaphorical simple epithets. This means that metaphorical epithets prevail in the example; therefore, we will analyze only them $(1-6)$. These epithets are organically included in a complex epithet design.

The epithet (6) iedzhegey badaraannaakh can literally be translated as "snow-white bog (just like) cottage cheese". The object is badaraan (a bog). The epithet iedzhegey (cottage cheese) is used in a figurative meaning, i.e. it acts as a metaphor. Such a technique was necessary for a more specific and figurative perception of the object. Therefore, this epithet is attributed to a simple metaphorical epithet. The following is an example (5) suumekh suduruunneekh (raindrops (just like) cottage curds), in which the object presents suduruun (raindrops). The epithet suumekh (cottage cheese curds) is expressed through a metaphor. Therefore, the analyzed epithet (5) can be attributed to a simple metaphorical epithet. The following example (4) hoyuu kymys kordugenneekh (a lake (just like) thick kumis). The object of the epithet is kordugen (a lake), the compound word hoyuu kumis (thick kumis) is a metaphor. As a result, we get a simple metaphorical epithet hoyuu kumis kordugenneekh (a lake (just like) thick kumis). In example (3), saaby kumis chalbakh (a puddle (just like) liquid kumis), the object is the word chalbakh (a puddle). The compound word saaby kumis 
(liquid kumis) is expressed in metaphor as an epithet. This expression can be figuratively translated as "snow-white puddles, like liquid kumis". Next epithet (2) horgholdzhun honuulaah (a meadow (just like) one made of tin). The object is honuu (a meadow). The word horgholdzhun (tin) is an epithet expressed through a metaphor. The next epithet (1) byrta myraannaah (a cape (just like) abdominal fat) consists of the object myraan (cape) and the metaphoric epithet byrta ((just like) abdominal fat). So, these examples are simple metaphorical epithets $(1-6)$ of the syhyy (valley) object (9).

As a result, a complex metaphorical epithet is formed, which gradually unfolds with a complex construction; this construction contains six simple metaphoric epithets. Therefore, the complex epithets with several attributes may contain several objects, each of which has its own epithets expressed by a metaphor.

Thus, the metaphoric epithets make up $15 \%$ of the general total of the analyzed epithets. They have both - a simple and a complex structure. The simple metaphoric epithets comprise of one object and one image. The complex epithets comprise of several objects, each of which has its own attributive combinations; these combinations include a noun in its direct meaning and a metaphor.

\section{Conclusion}

Epithets presented in the north-eastern Olonkho texts perform a descriptive and characterizing function. The majority of these epithets have a simple structure (74\%) which shows that language of the north-eastern Olonkho texts is simple and archaic in its nature. Olonkho epithets have a complex component structure. Complex epithets (26\%) are subdivided into three groups. The first group comprises of epithets with a comparison. The make up $45 \%$ of the general total of complex epithets. The second group consists of numerical epithets, which make up $40 \%$ of the overall total. We make the assumption that numerals contained in this type of epithet constructions had not only a comprehensive and descriptive but also an educational function. The third group is represented by metaphorical epithets, which amount for $15 \%$ of the overall total. In terms of their component structure, metaphoric epithets denote one object and one image. A distinctive feature of epithet constructions in the Olonkho-telling tradition overviewed in this paper is the presence of complex epithets with a few attributive components. A characteristic feature of these epithets is that one object and its several images contain simple metaphoric epithets. According to the thematic principle, metaphoric epithets are subdivided into five thematic groups.

The Olonkho-telling tradition typical for the Verkhoyansky region of Yakutia has the most complex epithet structure (36\% of complex epithets). This number is followed by the Momsky (29\%) and Abiysky (22\%) regions; complex epithets were found in the only existing complete version of Olonkho of these regions. The Olonkho about Kotor Mulgun ("Flying Mulgun") is an exception in this sequence as it only contains $14 \%$ of complex epithets.

We have concluded that among the overall total of epithets analyzed in Olonkho, the simplest epithet constructions are presented in the Srednekolymsky Olonkho-telling tradition. In this Olonkho, three Olonkho texts include $22 \%$ of complex epithets. It is worth mentioning that some types of Olonkho were not fully recorded on paper. Researchers recorded the retold versions of Olonkho from the Olonkho-storytellers. In one of the texts, the number of complex epithets makes up $61 \%$. 
Perhaps this resulted from the length of Olonkho and the quantity of selected epithets. The text mentioned above contains only 28 epithets which we were able to identify. Consequently, among the four local traditions mentioned in this paper, the Verkhoyansky region Olonkho have the most complex epithet structure; while the Srednekolymsky region Olonkho has the simplest epithet structure.

The complex epithets used in the north-eastern Olonkho-telling tradition play a significant role in the representation and perception as well as the creation of the esthetic potential of the Sakha people. The north-eastern Olonkho texts have a predominating number of simple epithets, which highlights the archaic character of the epic tradition reviewed in this paper.

\section{Acknowledgments}

This research was conducted with the financial support from the Russian Fund of Fundamental Research (RFFR) and was a part of a research project No.18-412-140013 p_a in the RFFR database.

\section{References}

Ammosov, K.H. (2008). Aalya, the Precise Bogatyr. Yakutsk: Bichik Publishing. (In Yakut language)

Archives of the Yakutsk Scientific Research Center SB RAS, Fund No.5.

Efimova, L. S. (2004). The lexical and stylistic features of the language used in round dance songs. Dissertation abstract. Yakutsk.

Ivanov-Kunde, A. A. (1979). The literary form of Yakut poetry: poetry and semantic. Yakutsk. (In Yakut language)

Izbekova, E. I. (2014). Numerals in Olonkho: structure and semantics. Yakutsk: NEFU Publishing House.

Muoma olonkholoro (Olonkho of Muoma) (2004). Ed. V.V. Illarionov. Yakutsk: Bichik.

Pavshuk, A. V. (2007). The linguistic nature and functions of epithets in a literary text: the novel "Willpower" by Asorin as a case-study. Dissertation abstract. Moscow.

Petrov, V. T. (1978). Folklore traditions in Yakut literature of the Soviet period. Moscow: Nauka.

Pukhov, I. V. (1962). The Yakut heroic epic Olonkho. The main images. Moscow: The Academy of Science of the USSR Publishing House.

Romanova, L. N. (2002). A. E. Kulakovsky and his contemporaries: special features of poetic language), Novosibirsk: Nauka Press.

Selivanov, F. M. (1977). The poetic structure of bylinas. Moscow.

Semenova, V. G. (1999). The poetic universe of Anampodist Sofronov (Sources. Issues. Poetics). Dissertation abstract. Yakutsk.

Sleptsov, P. A. (1989). Yakut olonkho: vocabulary, semantics. Yakutsk: Yakutsk Scientific Research Center of the USSR SB RAS.

Tomashevsky, B. V. (2002). Theory of Literature. Poetics. Moscow.

Veselovsky, A. N. (1940). Historical Poetics. Leningrad. 